\title{
Raman Scattering by Bulk Phonons in Microcrystalline Silver Via Electronic Surface Excitations
}

\author{
W. Akemann ${ }^{*}$, A. Otto ${ }^{a}$, and H. Schober ${ }^{b}$ \\ ${ }^{a}$ Lehrstuhl für Oberflächenwissenschaft, Heinrich-Heine-Universität Düsseldorf \\ 40225 Dïsseldorf, FR Germany, EU \\ ${ }^{b}$ Institut für Festkörperforschung, Forschungsanlage Jülich, \\ 52425 Jülich, FR Germany, EU
}

Received: July 17, 1996

\begin{abstract}
A assim chamado espalhamento de fundo inclástico, de filmes metálicos obtidos a partir da condensação de metais nobres em temperaturas criogênicas, foi recentemente atribuído à excitação de pares elétron-lacuna na banda referente ao estado de superficie tip Schockley em facetas (111). Este mecanismo requer rugosidade superficial. A aniquilação de pares elétron-lacuna tipo Schockley por espalhamento de fônons dá origem a um espectro Raman de fồnons. Uma boa concordância é obtida com espectros teóricos baseados em dados de espalhamento de nêutrons. $O$ aparecimento do espectro de fônons com o recozimento entre 120 e $250 \mathrm{~K}$ corresponde ao crescimento no tamanho médio das facetas (111) de 4,8 a $7 \mathrm{~mm}$. Nỏs conjecturamos que os estados superficiais de Schockley em superfícies não recobertas são substituídos, no caso de sistemas aquosos contendo eletrólitos, pela ressonância de Newns-Anderson próxima ao nível de Fermi induzida pelo haleto, e que um mecanismo análogo de excitação de elétrons de superfície explica o espectro de fônons observado em tais condições.

The so-called inelastic background of clean cryocondensed noble metal films was recently ${ }^{8}$ assigned to excited electron hole pairs in the Shockley surface state band of (111) facets. This mechanism needs surface roughness. Annihilation of the Shockley e-h pairs by scattering from phonons leads to a Raman spectrum of phonons. Good agreement is achieved with theoretical spectra based on data from neutron scattering. The development of the phonon spectra with annealing from 120 to $250 \mathrm{~K}$ corresponds to the growth of the average size of (111) facets from 4.8 to $7 \mathrm{~nm}$.

We conjecture that the Shockley surface states at uncovered surfaces are replaced by halogeninduced Newns-Anderson resonance near the Fermi level in aqueous halide electrolytes and that an analogous mechanism of electron surface excitations explains the phonon signal under these conditions.
\end{abstract}

Keywords: SERS, electronic surface excitations, phonons

\section{Introduction}

The Raman spectra of silver substrates used in surface enhanced Raman spectroscopy (SERS) (e.g. cryocondensed silver films grown on a smooth substrate at low temperature in ultrahigh vacuum or silver electrodes roughened by an oxidation-reduction cycle in electrolyte solutions) are known to show broad, but clearly resolvable, low frequency bands at about $65 \mathrm{~cm}^{-1}, 110 \mathrm{~cm}^{-1}$, and $170 \mathrm{~cm}^{-1}$ (see for instance Ref. 1 and references therein). The bands were first observed by Pockrand and $\mathrm{Otto}^{2}$ who realized that the frequencies of the spectral features coincide with critical points in the phonon band structure of bulk silver ${ }^{3}$. Consequently, they assigned the bands to disorder induced light scattering by lattice phonons ${ }^{4}$. This model, however, was objected to by other authors who proposed that the bands are due to local modes of small silver aggregates on top of the surface ${ }^{5,1}$. Specifically, Roy and Furtak ${ }^{6}$ worked out in detail a model that implies the existence of a majority of a surface species with well-defined pyramidal $\mathrm{Ag}_{4}$ struc- 
ture. Roy and Furtak suggested that cationic $\mathrm{Ag}_{4}$ clusters might represent the sites of maximum enhancement. Because of the controversial assignments, we found it necessary to give a new account of the low frequency SER spectra of silver.

\section{Experimental Results and Discussion}

Following the work of Pockrand and Otto ${ }^{2 *}$, we prepared silver films by thermal evaporation onto a polished copper substrate within UHV. In our case, the substrate temperature was $40 \mathrm{~K}$ and the final film thickness was 150 $\mathrm{nm}$. For an in situ recording of the Raman spectra we used cw $514.5 \mathrm{~nm}$ laser radiation of $0.1 \mathrm{~W}$ power, conventional optics and a triple monochromator (see Ref. 7 for details).

In Fig. la we have compiled the low frequency spectra of a silver film for various temperatures of annealing. To this end, we heated the film to increasing temperatures, $T$, for $120 \mathrm{~s}$ and recorded each spectrum after recooling to $40 \mathrm{~K}$. In a previous publication ${ }^{8}$ we discussed the influence of the annealing procedure on the Raman spectra in the range above $250 \mathrm{~cm}^{-1}$ where the spectra show a broad continuum of electronic excitations. It was shown that thermal annealing smoothens the surface structure by inducing the growth of low-index facet planes and decreasing the atomic defect density. In Fig. la it can be seen that annealing from $120 \mathrm{~K}$ to $250 \mathrm{~K}$ considerably increases the overall scattering intensity, while further annealing to 350 $\mathrm{K}$ causes an almost complete loss of inelastically scattered light, leaving only the dark current to be detected beyond $\pm 20 \mathrm{~cm}^{-1}$. This demonstrates the capability of our spectrometer to suppress spurious light of zero order for Stokes frequencies above $20 \mathrm{~cm}^{-1}$. Two observations are of particular importance in this context: first, the three bands at $68 \mathrm{~cm}^{-1}, 112 \mathrm{~cm}^{-1}$, and $166 \mathrm{~cm}^{-1}$ appear to be superimposed on a continuous spectrum of scattered light, with the intensity increasing from $180 \mathrm{~cm}^{-1}$ towards lower wavenumbers (note the logarithmic intensity scale). Second, the bands are not altered in shape or spectral position as the film is annealed. It is simply the overall intensity of the spectrum that responds to the annealing treatment. Interestingly, a similar observation was reported in the case of silver electrodes in an aqueous $\mathrm{Cl}^{-}$electrolyte. Macomber and Furtak ${ }^{9}$ observed that bands at $110 \mathrm{~cm}^{-1}$ and $160 \mathrm{~cm}^{-1}$ loose intensity when the electrode is heated from $296 \mathrm{~K}$ to $313 \mathrm{~K}$ while kept at $-0.3 \mathrm{~V}$ (SCE). According to Roy and Furtak ${ }^{6}$ this is due to an annealing-induced loss of active sites which they claim obeys the $\mathrm{Ag}_{4}$ structure. The same argument in the present case would imply that the number of $\mathrm{Ag}_{4}$ clusters increases upon annealing to $250 \mathrm{~K}$, and only decreases for higher temperatures. By the formation of $\mathrm{Ag}_{4}$ from smaller precursor clusters below $250 \mathrm{~K}$, and by an agglomeration of $\mathrm{Ag}_{4}$ clusters above $250 \mathrm{~K}$. However, we do not see why
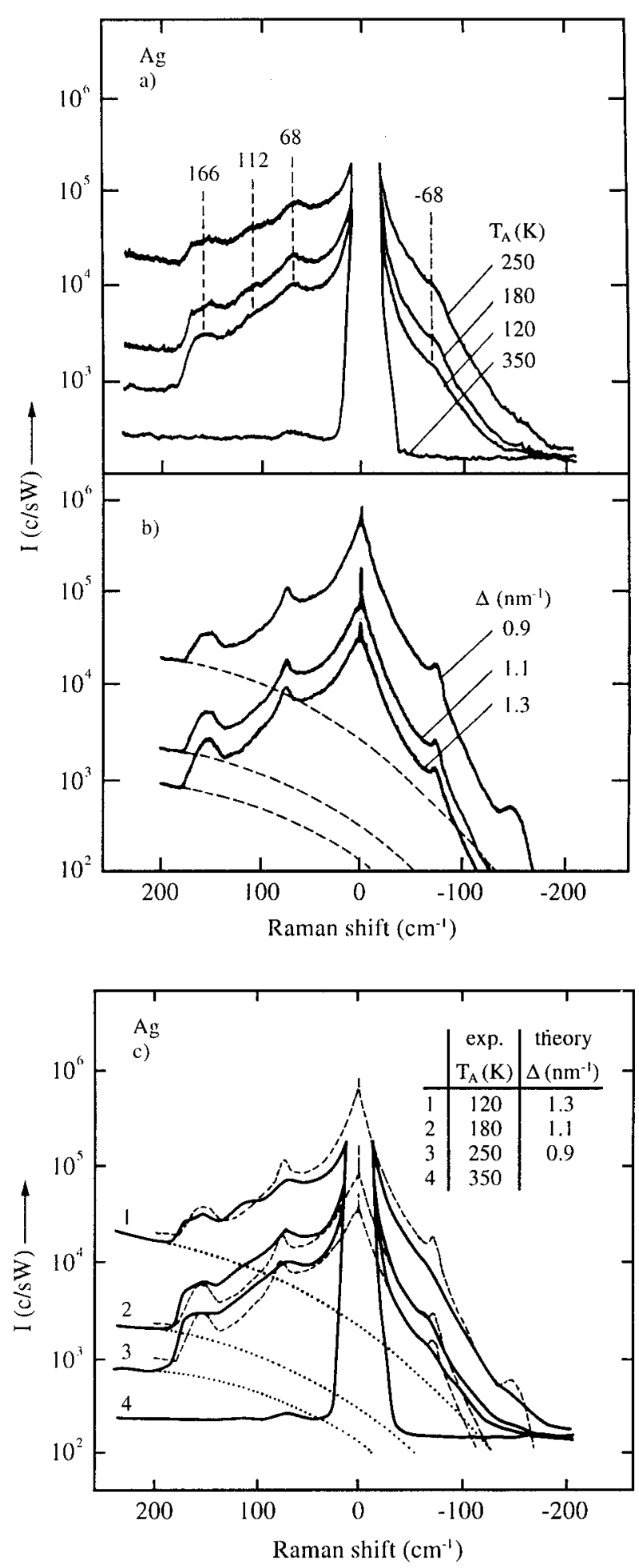

Figure 1. a) Low frequency Raman spectra of a silver film that was cryocondensed at $40 \mathrm{~K}$ and subsequently annealed at the indicated temperatures, $T_{A}$, for about $120 \mathrm{~s}$. All spectra were recorded at a common temperature of $40 \mathrm{~K}$. b) Theoretical Raman scattering spectra as a sum (solid line) of the bulk phonons projected on (111) and the intra-Shockley surface band transitions (dotted lines), see text. Parameters from bottom to top: $\Delta=1.3,1.1,0.9 \mathrm{~nm}^{-1}$. c) A comparison of experimental (solid lines) and theoretical (dashed) spectra, see text. 
the Raman spectrum should be sensitive only to $\mathrm{Ag}_{4}$ and not to $\mathrm{Ag}_{\mathrm{n}}(\mathrm{n} \geq 1)$ in general.

Another important experiment to test the cluster assignment is to look for the influence of adsorbates. We investigated oxygen in particular because it is known that oxygen does not chemisorb at the smooth (111) facet planes of silver at $40 \mathrm{~K}$, but only adsorbs dissociatively at "surface defects" ${ }^{10}$. In the SERS spectrum of Fig. 2, the band centered at $340 \mathrm{~cm}^{-1}$ is assigned to an $\mathrm{Ag}-0$ stretch vibration and thus indicates atomic oxygen. No bands indicative of a molecular oxygen species ${ }^{11}$ are observed. The continuous background of electronic Raman scattering between $250 \mathrm{~cm}^{-1}$ and $3000 \mathrm{~cm}^{-1}$ uniformly drops upon the adsorption of oxygen. If a major portion of the "defects" obeyed an $\mathrm{Ag}_{4}$ structure, one would have to assume that oxygen attaches to the cluster sites, and thus an $\mathrm{Ag}_{4} \mathrm{O}_{\mathrm{x}}$ species would be created at the surface, with $x$ probably between 1 and 2 . If the correct assignment of the low frequency bands was the vibrations of the $\mathrm{Ag}_{4}$ clusters, a shift of frequencies due to a loading of the clusters with oxygen atoms should be observed. However, in Fig. 3 we observe a uniform decline in intensity, with the position of the bands fixed.

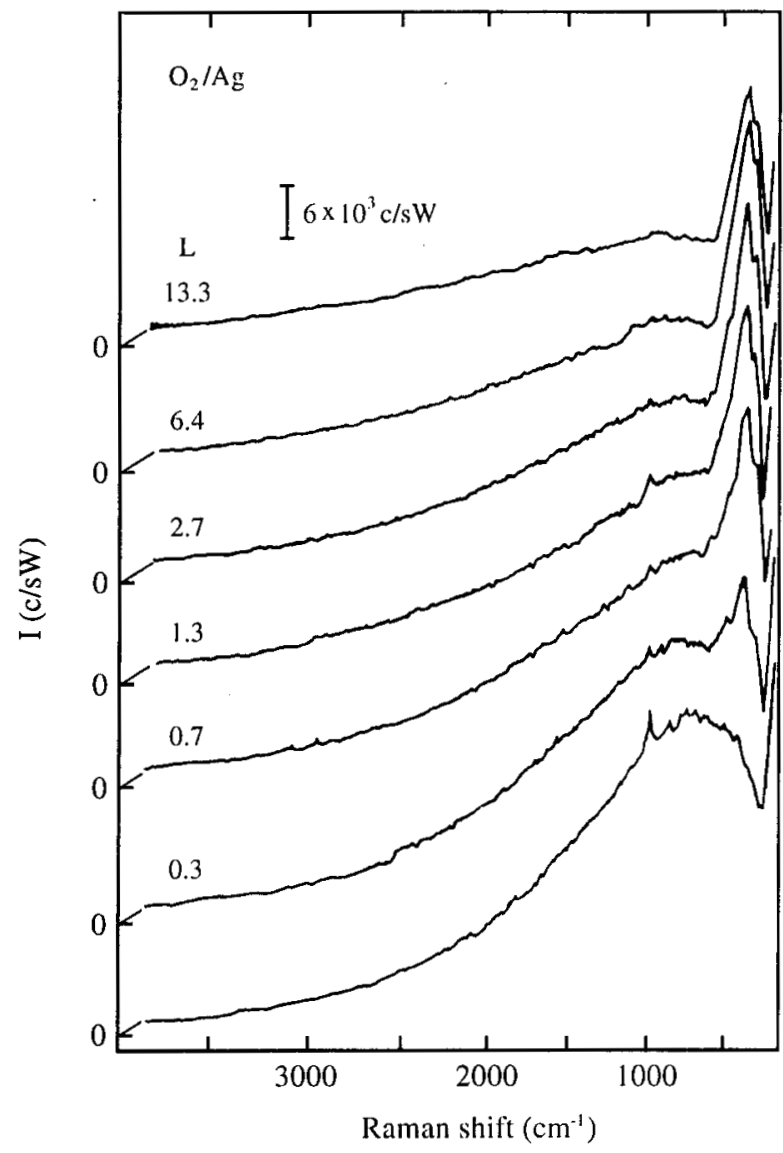

Figure 2. Raman spectra from $200 \mathrm{~cm}^{-1}$ to $3800 \mathrm{~cm}^{-1}$ of a silver film that was cryocondensed at $40 \mathrm{~K}$, then annealed at $250 \mathrm{~K}$, and finally exposed to oxygen at $40 \mathrm{~K}$. The exposures are quoted in Langmuir (L).
We believe that this behavior is in contradiction to what would follow from the cluster assignment. Again, we refer to the work of Roy and Furtak ${ }^{6}$ who comment on the observation that the frequencies of the bands from a silver electrode neither depend on the nature of the specifically adsorbed anions, e.g. $\mathrm{Cl}^{-}, \mathrm{Br}^{-}$or $\mathrm{J}^{-}$(see also Ref. 5), nor on their density of coverage ${ }^{6}$, which is a function of the applied electrode potential. The authors assume that the anions, for example $\mathrm{Cl}^{-}$, are adsorbed at the silver surface with a minimum distance of $0.6 \mathrm{~nm}$ from the $\mathrm{Ag}_{4}{ }^{+}$cluster sites and that the interaction of anionic and cluster species is hence only by virtue of the dipolar electrostatic fields deriving from the ionic and image charges. Calculating within this framework, the harmonic cluster potential in the presence of the adsorbed anions, Roy and Furtak conclude that the anions should not significantly affect the cluster vibrational frequencies.

However, the situation here is different. Since oxygen would anionically bond to cluster atoms, as argued above, it is obvious that the adsorption of oxygen would cause a redistribution of the valence electronic charge between the cluster atoms. Thus we would expect new frequencies in disagreement with what is observed. Altogether our results provide no strict evidence recommending the cluster assignment. Instead, bulk phonons would easily explain the facts. Evidently, no alteration of frequencies is expected to result from substrate annealing, adsorption, or any other kind of surface manipulation in this case. Furthermore, the phonon assignment is supported by the observation that other metals display similar bands, for example copper ${ }^{12,13}$, at $105 \mathrm{~cm}^{-1}, 160 \mathrm{~cm}^{-1}$, and $220 \mathrm{~cm}^{-1}$, and potassium ${ }^{14}$ at $50 \mathrm{~cm}^{-1}, 70 \mathrm{~cm}^{-1}$, and $80 \mathrm{~cm}^{-1}$. In each case the observed bands center at frequencies of van Hove singularities in the phonon density of states ${ }^{15}$. The quenching of the intensity by oxygen adsorption, and the variation of the intensity

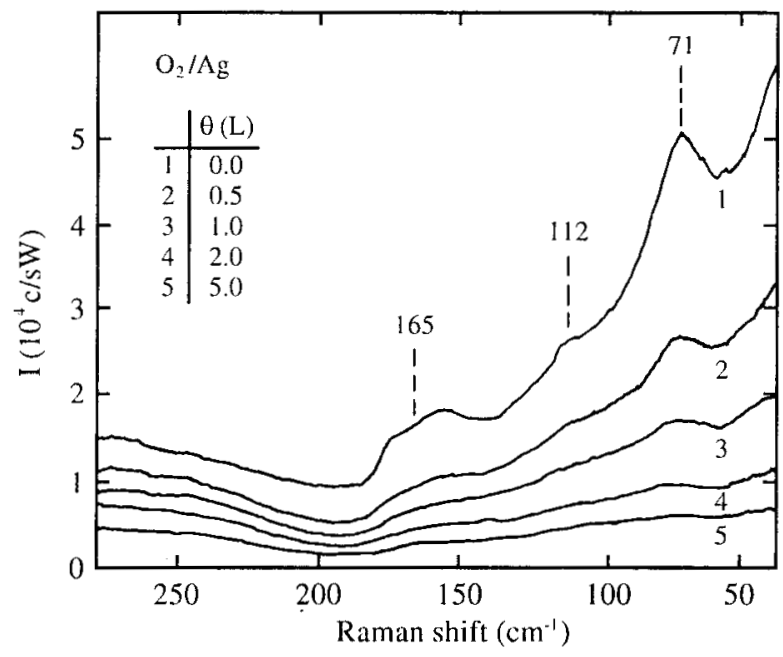

Figure 3. Low frequency Raman spectra of a silver film after being annealed at $250 \mathrm{~K}$ and exposed to increasing doses of oxygen at $40 \mathrm{~K}$. 
with the electrochemical potential in the case of electrode surfaces ${ }^{16}$, indicate a surface sensitive mechanism of excitation. There will be little enhancement of the laser field within the metal, because the enhancement of the local macroscopic field by surface plasmon type resonances is restricted mainly to the field outside the metal, normal to the local surface ${ }^{17}$. Therefore Raman scattering by phonons within the penetration depth of visible light is expected to be weak. Furthermore, a quenching of the Raman intensities by a decrease of the local macroscopic electromagnetic field should be connected with a change in the optical absorption. However, this is not observed ${ }^{18}$.

In the following section, we propose a mechanism for clean films in UHV similar to our explanation of the electronic Raman scattering above $200 \mathrm{~cm}^{-1}$ (see Fig. 2) as given in Ref. 8. A possible extension of this model to the electrode surface in aqueous halide-containing electrolytes, such as in the work of Roy and Furtak ${ }^{1,6}$, is discussed in the Open Questions and Conjectures section.

\section{Raman scattering by bulk phonons via electronic surface excitations}

Recently it has become obvious that electronic surface states at metal surfaces ${ }^{19}$ influence surface reactions ${ }^{20}$. So-called s/p derived crystal-induced surface states may penetrate several lattice constants into the bulk. For instance, the Shockley surface state at $\mathrm{Ag}(111)$ surfaces $^{21}$ has a decay length of $2.8 \mathrm{~nm}$ into the bulk, as determined experimentally ${ }^{22}$. An electron in this state will therefore scatter not only from surface phonons but also from bulk phonons.

The proposed Raman scattering from phonons involves virtual transitions between electronic surface states at a rough surface. Most likely, the surface of a cryocondensed and partly annealed film consists of small facets of (111), (110), and (100) orientations, various steps, kinks and other one- and zero-dimensional defects related to grain boundaries and dislocations.

Electronic surface states, well known from large single crystal surfaces ${ }^{23}$, still exist on small facets; for instance the Shockley $\mathrm{Au}(111)$ state was observed as spatially confined on terraces with widths in the range of $3 \mathrm{~nm}$ to $6 \mathrm{~nm}$ and islands of a $4.5 \mathrm{~nm}$ diameter ${ }^{24}$ and the first image state on $\mathrm{Cu}(100)$ with step spacings of $1 \mathrm{~nm}^{25,26}$ and on monolayer $\mathrm{Ag}$ islands on Pd(111) with diameter $1-10 \mathrm{~nm}^{27}$.

The optical transition between the Shockley state and the image states on $\operatorname{Ag}(111)$ is only excited by p-polarized $\operatorname{light}^{28}$. One might also expect an excitation by s-polarized light at a stepped (111) surface and the electrical vector perpendicular to the steps, but no experimental investigation is known ${ }^{29}$.

The physical scattering mechanism is given as a 4th order process, involving photon annihilation by electronic excitations from the occupied part of the Shockley surface state band $\left(S_{2}\right.$ in Fig. 4$)$ to image states ( $S_{1}$ in Fig. 4), photon creation by electronic transition from image states to the unfilled part of the Shockley surface state band, scattering within the band of image states by surface roughness, and scattering within the Shockley band by phonons. Also the scattering within the Shockley band by surface roughness and within the image state bands by phonons contributes. The resonant process among the 24 different time ordered processes is schematically given in Fig 4 . The analogy to Raman scattering in disordered semiconductors, involving valence and conduction bands, and intraband scattering by disorder and phonons, is evident. An alternative or additional process may involve the electronic transition between the Shockley state and the bulk states above $\mathrm{L}_{1}$ (see Fig. 4). These transitions have been discussed in the context of second harmonic generation (SHG) ${ }^{30}$.

A laser wavelength of $514.5 \mathrm{~nm}$ is far out of resonance with the transition between the Skockley and the first image state at $318 \mathrm{~nm}^{31}$ or the bulk states above $L_{1}$. Out of resonance, in the case of Raman scattering of vibrations of molecules and bulk crystals, the polarizability theory of Raman scattering by G. Placzek is usually used (see Ref. 32 ), with the assumption of homogeneous laser and Raman fields (frequency $\omega_{\mathrm{L}}$ ) and the same derivative of the atomic polarizability with respect to displacement from the equilibrium conditions. This is not possible in our case, because the nonlocal electromagnetic fields are highly inhomogeneous near the surface and the electronic surface states are localized near the surface.

Nevertheless, one may define an electronic surface susceptibility, $\chi$ modulated by lattice vibrations with normal coordinates, $\xi$. Analogous to Eqs. 2.46 and 2.50 in Ref. 32 for first order Raman scattering (s: Stokes, as: antiStokes) by phonons in crystals (frequency $\omega_{\mathrm{ph}}$ ), we derive for the first order Raman scattering at crystalline surfaces via electronic surface states:

$$
\begin{array}{ll}
\omega_{\mathrm{S}}=\omega_{\mathrm{L}}-\omega_{\mathrm{ph}}, & \omega_{\mathrm{as}}=\omega_{\mathrm{L}}+\omega_{\mathrm{ph}} \\
\underline{\mathrm{k}}_{\mathrm{s}}=\underline{\mathrm{k}}_{\mathrm{L}}-\underline{\mathrm{q}}, & \underline{\mathrm{k}_{\mathrm{as}}}=\underline{\mathrm{k}}_{\mathrm{L}}+\underline{\mathrm{q}}
\end{array}
$$

where the k-vectors are 2-dimensional projections of the 3-dimensional wave vectors of the incident laser and emitted Stokes and anti-Stokes fields onto the surface, and $q$ is the 2-dimensional wavevector of the lattice vibrations parallel to the surface.

The differential Stokes and anti-Stokes power scattering cross sections are given, respectively, by Eqs. $2 \mathrm{a}$ and $2 \mathrm{~b}$ :

$$
\begin{aligned}
\frac{d \sigma_{s}}{d \Omega}(\xi)=\frac{\omega_{s}^{4}}{(4 \pi)^{2} c^{4}} F \mid \underline{e}_{s} & \left.\frac{d \chi}{\underline{d \xi}} \underline{e}_{L}\right|^{2} \\
& \frac{\hbar}{2 \omega_{p h}(\xi)}(n(\xi)+1)
\end{aligned}
$$



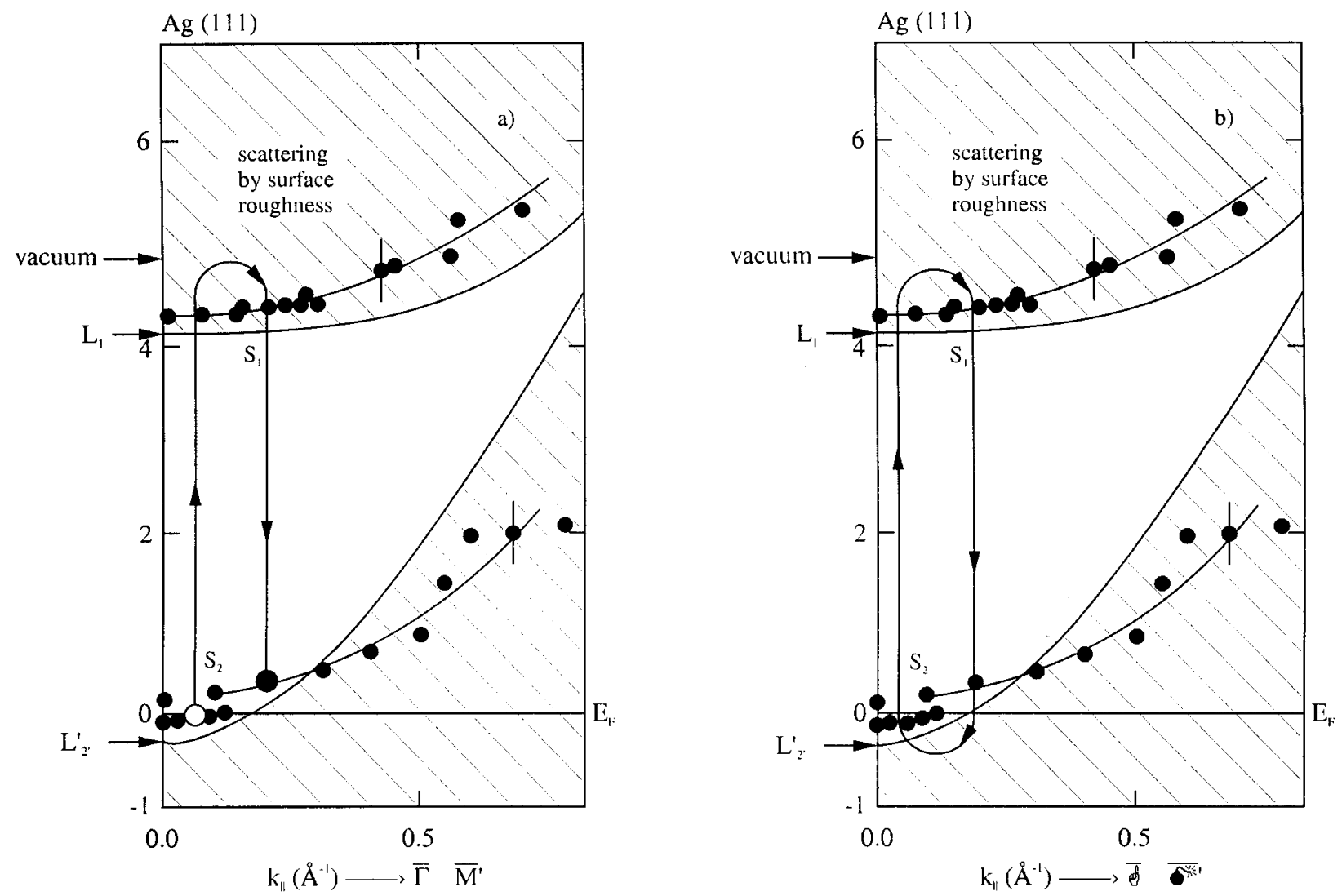

Figure 4. The mechanism of Raman scattering by excitation of electron-hole pairs in the Shocklcy band $\mathrm{S}_{2}$ (a) and of phonons (b) via coupling to surface states. $S_{1}$ is the lowest image state. The experimental data of $S_{1}$ and $S_{2}$ are from Ref. 60 . According to 2-photon photocmission and $S H G$, the first image state is found $3.84 \mathrm{eV}$ above the Shockley state at $\mathrm{k}=0^{61}$.

$$
\frac{d \sigma_{a s}}{d \Omega}(\xi)=\frac{\omega_{a s}^{4}}{(4 \pi)^{2} c^{4}} F\left|\underline{e}_{a s} \frac{d \chi}{\underline{\underline{d \xi}}} \underline{e}_{L}\right|^{2} \frac{\hbar}{2 \omega_{p h l}(\xi)} n(\xi)
$$

Here $\mathrm{e}_{s}, \mathrm{e}_{\mathrm{as}}$ and $\underline{\mathrm{e}}_{\mathrm{L}}$ are the macroscopic Stokes, antiStokes, and laser fields above the surface, by the choice of the definition of the surface susceptibility, $\mathrm{n}(\xi)$ is the BoseEinstein factor of a phonon normal mode with normal coordinate $\xi$ in harmonic approximation, and $F$ is the illuminated surface area.

The results, which we will use in the following are Eq. $1 \mathrm{~b}$ which is a consequence of the translational symmetry of the local surface, and the factors $(\mathrm{n}(\xi)+1) / \omega_{\mathrm{ph}}(\xi)$ and $\mathrm{n}_{\mathrm{ph}}(\xi) / \omega_{\mathrm{ph}}(\xi)$, which are consequences of the quantization of a harmonic oscillator.

For an elemental crystal with acoustic lattice vibrations only, $\mathrm{g}$ is very small due to Eq. $1 \mathrm{~b}$, and consequently inelastic light scattering from phonons may only be observed by Brillouin scattering. This is different when the surface roughness allows for elastic scattering by a 2 dimensional vector, $\mathrm{k}_{\mathrm{r}}$. Then, Raman scattering becomes possible for phonons with wavevector $\mathfrak{q}=-\mathrm{k}_{\mathrm{r}}$.

We assume two dimensional isotropic dispersion of the Shockley band and a constant tensor, $d \chi / d \xi$, for all pho- nons. We consider only bulk phonons, the relation $\omega_{\text {ph }}(Q)$ for wavevectors $Q$ throughout the bulk Brillouin zone is obtained by a Born-von Karmann $\mathrm{fit}^{15}$ to the experimental phonon dispersion along high symmetry directions. We have chosen the data of Kamitakahara and Brookhouse ${ }^{3}$ rather than those of Drexel ${ }^{33}$, because the latter show a strong deviation from the experimental data at the $\mathrm{W}$ point of the Brillouin zone.

The scattering probability by surface roughness is modeled by a Lorentzian distribution:

$$
S(\underline{k},)=\frac{2 \Delta}{\pi} \frac{1}{\Delta^{2}+\underline{k}^{2}}
$$

$q$ is the projection of $Q$ onto the (111), (110), or (100) plane as given, respectively, by:

$$
\begin{aligned}
& |q|=\left(\left(Q^{2}-1 / 3\left(Q_{x}+Q_{y}+Q_{z}\right)^{2}\right)^{1 / 2}\right. \\
& |q|=\left(\left(Q^{2}-1 / 2\left(Q_{x}+Q_{y}\right)^{2}\right)^{1 / 2}\right. \\
& |\mathrm{g}|=\left(Q_{x}{ }^{2}+Q_{y}{ }^{2}\right)^{1 / 2}
\end{aligned}
$$

The Stokes and anti-Stokes phonon spectra are given by Eqs. $5 \mathrm{a}$ and $5 \mathrm{~b}$ : 


$$
\begin{aligned}
& J_{s, p h}(\omega) \propto \frac{n(\omega)+1}{\omega} \frac{1}{3 N} \sum_{Q j} S(q) \delta\left(\omega-\omega_{p h}(Q, j)\right) \\
& J_{a s, p h}(\omega) \propto \frac{n(\omega)}{\omega} \frac{1}{3 N} \sum_{Q j} S(q) \delta\left(\omega-\omega_{p h}(Q, j)\right)
\end{aligned}
$$

where the index $j$ runs over the 3 acoustic branches.

The integrals in Eq. 5 were evaluated with the GilatRaubenheimer method ${ }^{34}$. Fig 5 shows that the projection of the bulk phonon structure on the three low index faces yields significant and individual structure.

To the Raman spectrum of the phonons projected on (111), we add the electronic Raman spectra of the intraShockley band transitions at (111) facets, as given by:

$$
\begin{aligned}
J_{e h, s h}(\omega) \propto \int f\left(\varepsilon_{s h}\left(\underline{k}_{s h}\right)\right) S\left(k_{h}\right)\left(1-f\left(\varepsilon_{s h}\left(\underline{k}_{s h}+\underline{k}\right)\right)\right) \cdot \\
\cdot \delta\left(\omega-\varepsilon_{s h}\left(\underline{k}_{s h}+\underline{k}_{h}\right)+\varepsilon_{s h}\left(\underline{k}_{s h}\right)\right) d \underline{k}_{s h} d \underline{k}_{r} .
\end{aligned}
$$

where $\omega$ is taken as positive on the Stokes side and negative on the anti-Stokes side, and $f(\omega)$ is the Thomas-Fermi distribution. The quadratic dispersion of the Shockley band was modeled with the experimental data ${ }^{35}$ at $65 \mathrm{~K}$ ( $\mathrm{E}_{\mathrm{sl}}\left(\mathrm{K}_{\mathrm{sl}}\right.$ $=0)-\mathrm{E}_{\mathrm{F}}=-60 \mathrm{meV}$ and $\mathrm{m}_{\mathrm{eff}} / \mathrm{m}=0.43$ ) by:

$$
\frac{E_{s h}-E_{F}}{\hbar}=\varepsilon_{s}=-\varepsilon_{0}+\frac{\hbar \underline{k}_{s h}^{2}}{2 m_{e f f}}
$$

We neglect the intra surface band transitions at the (110) and (100) facets in comparison with the experimental data because these will deliver a considerably weaker signal. The intensities will depend on the number of occupied surface states per unit area of the different surface orientations. Since not all data for silver are available ${ }^{36}$, we use those of $\mathrm{Cu}$ compiled in Ref. 37. By assuming two dimensional isotropic dispersion of the occupied surface states near the $X$ point of the (100) surface Brillouin zone, the $Y$ point of the (110) surface Brillouin zone, and the $\Gamma$ point of the (111) surface Brillouin zone, the number, n, of electrons in the surface state per surface atom is given by

$$
\mathrm{n}=\frac{2 \mathrm{~m}_{\mathrm{eff}}}{\hbar^{2}}\left(\mathrm{EF}_{\mathrm{F}}-\mathrm{E}_{0}\right) \frac{\mathrm{A}}{2 \pi}
$$

where $m_{\text {eff }}$ is the effective mass of the surface band (which has the lowest energy $E_{0}$ at the $X, Y$, or $\Gamma$ point, respectively), $E_{F}$ is the Fermi energy and $A$ is the area of the primitive surface unit cell, depending on the orientation of the surface. With Eq. 8 and the experimental values of $m_{\mathrm{eff}}$ and $E_{F}$ in Table 1 of Ref. 37 we obtain:

$$
\mathrm{n}(100)=0.010, \mathrm{n}(110)=0.0407, \mathrm{n}(111)=0.0424
$$

A detailed calculation in the sense of Eq. 6 for copper yields a ratio of the intensities of intra-surface band transitions at $(111):(110):(100)$ of $1: 0.33: 0.03^{38}$. On $\mathrm{Ag}(110)$ a resonant contribution is expected at a laser photon energy

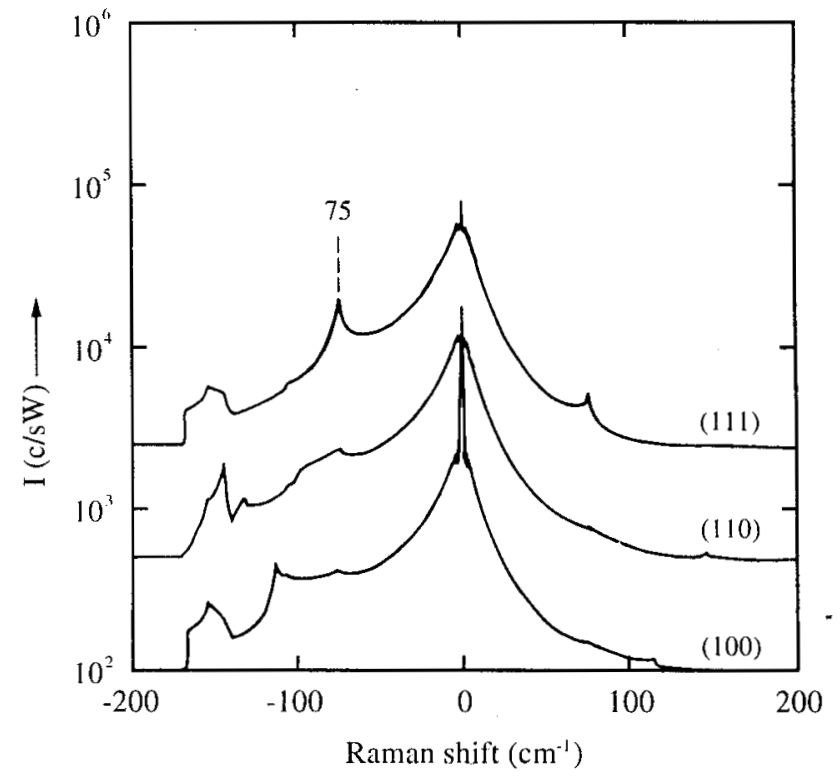

Figure 5. Theoretical Raman scattering spectra of the bulk phonons of silver (data from Kamikatahara and Brookhouse ${ }^{3}$ ) according to Eqs. 4 and 5 for $\Delta=1.1 \mathrm{~nm}^{-1}$ with arbitrary units of intensity.

of $1.75 \mathrm{eV}$ because of a transition between an occupied and an unoccupied crystal-induced surface state, as observed by second harmonic generation ${ }^{39}$. A laser photon energy of $2.41 \mathrm{eV}$ is well outside of this resonance. We also expect that the (111) facets are more abundant than the (110) facets, particularly when annealing to a temperature of $250 \mathrm{~K}$.

There remain two parameters for a fit to the experimental spectra using the bulk phonon structure and the intrasurface band transitions at (111) facets, namely the width, $\Delta$, of the roughness spectrum and the ratio of the prefactors of $\mathrm{J}_{\mathrm{s}, \mathrm{ph}}$ or $\mathrm{J}_{\mathrm{as}, \mathrm{ph}}$ and $\mathrm{J}_{\mathrm{as}, \mathrm{ph}}$, independent of the choice of $\Delta$.

A relatively good fit (considering an error of the phonon frequencies obtained by neutron scattering in non symmetry directions of $Q$ of 5-10\%, electron phonon interaction and anharmonic effects) is obtained by calculating an average spectrum by random variations in the values of $\omega_{\text {ph }}(Q, j)$ within $5 \%$ (see Fig. 1b). The values of $\Delta$ imply that the average (111) facet size grows from 4.8 to $7.0 \mathrm{~nm}$ by annealing the films from 120 to $250 \mathrm{~K}$.

The small deviations from the experimental spectra at about 166 and $112 \mathrm{~cm}^{-1}$ (see Fig. 1c) are assigned to a weak contribution from (110) facets. We expect a contribution from the Rayleigh phonons of $\mathrm{Ag}(111)^{40,41}$ as well. The experimental values are $68.5 \mathrm{~cm}^{-1}$ and $73.4 \mathrm{~cm}^{-1}$ at the Mand K-point of the (111) surface Brillouin zone, respectively. These surface phonons probably contribute to the experimental structure at $68 \mathrm{~cm}^{-1}$ in Fig. la, but are not separated from the bulk phonon signal due to the small differences in the energy of the bulk and Rayleigh phonons at the M-point t! $^{\text {. }}$ 
The theory does not yield a peak at very low frequencies of $10-20 \mathrm{~cm}^{-1}$, in agreement with experiments described in Ref. 42. A more quantitative evaluation would require experiments on well-characterized vicinal faces.

\section{Open Questions and Conjectures}

The surface electronic states are altered by adsorption, and therefore the above model must be generalized. For instance, the photoemission spectrum of $\mathrm{Cu}(111)$ no longer displays the peak due to the Shockley state after the adsorption of $\mathrm{CO}^{43}$. Nevertheless, the Raman spectrum of colddeposited copper displays the characteristic structure, assigned to phonons, also unchanged after $\mathrm{CO}$ exposure ${ }^{12}$.

The Raman spectra from roughened silver electrodes in halide solutions (Ref. 1 and references therein) show besides the halide-silver vibrational band features at 70,110 and $160 \mathrm{~cm}^{-1}$, similar to the spectrum from clean cold-deposited films (see the Introduction). Of course, the Shockley state at a clean surface will not survive the halide adsorption. Angle resolved photoemission spectra of chlorine on $\mathrm{Cu}(111)^{44}$ show that the Shockley surface state vanishes with small amounts of $\mathrm{Cl}$. After the formation of the $\sqrt{3} \times \sqrt{3} \quad R 30^{\circ}-C l$ structure, the spectra display a narrow $\mathrm{Cl} 3 p$ derived antibonding surface band $2.3 \mathrm{eV}$ below the Fermi level. However, the electronic structure is different at an electrode surface. Though the $\sqrt{3} \times \sqrt{3} \quad \mathrm{R}_{3} 0^{\circ}-\mathrm{Cl}$ structure has also been found on $\operatorname{Ag}(111)$ at an anodic potential above $0.1 \mathrm{~V}_{\mathrm{Ag} / \mathrm{AgCl}}{ }^{45}$, the chlorine is neutral and it takes a transfer of one electron per atom to desorb it as a chloride ion ${ }^{46}$. This structure and the electrosorption valency of -1 of chloride is corroborated by $\mathrm{X}$-ray diffraction of chloride at $\mathrm{Au}(111)$ electrodes ${ }^{46}$. The eventual full discharge of specifically adsorbed $\mathrm{Cl}^{-}$at $\mathrm{Ag}(110)$, with an increasingly positive charge of the electrode surface may be inferred from Ref. 47. We conclude, following the dissertation by $\mathrm{H}$. Winkes ${ }^{48}$, that there must exist a Cl-induced resonance of the density of states at the Fermi level in the sense of the Newns-Anderson mode ${ }^{49}$, in contrast to the case of the silver-UHV interface. The neutral state of $\mathrm{Cl}$ is stabilized by the static field of the hydrated $\mathrm{Cl}^{-}$anions in the diffuse layer. The shift of the Newns-Anderson resonance towards the Fermi level is also indicated by the increasing electrical resistance of thin $\mathrm{Ag}(111)$ films in an aqueous $\mathrm{Cl}^{-}$electrolyte at an increasingly positive potential $^{48}$. Interestingly, the intensities of the halide-silver vibrational band and the phonon structure increase in the same way as the negative electrosorption valency of specifically adsorbed chloride, and also like the surface resistivity, as inferred by inspection of the results in Refs. 1, 16, 50 , and 51.

Therefore, it is likely that the filled Shockley state at a clean surface just below $\mathrm{E}_{\mathrm{F}}$ of about 0.04 electrons per surface atom is replaced by a halide resonaice, which centers at $\mathrm{E}_{\mathrm{F}}$ and contains 0.33 electrons per surface atom when the $\sqrt{3} \times \sqrt{3} \quad R 30^{\circ}-C l$ structure at the $\mathrm{Ag}(111)$ electrode is complete.

Potassium has no crystal-derived surface states, but displays the "phonon structure" in the Raman spectra of cold-deposited films ${ }^{14}$.

If we want to maintain the model discussed also for covered surfaces, we have to postulate that the surface susceptibility, $\chi$, is not considerably changed by physisorbates and is also present at a potassium surface. At electrode surfaces in halide electrolytes the halogen resonance at the Fermi level takes the role of the Shockley states at the uncovered surface. The optical surface excitations in this case are not yet known, but in the case of adsorbed bromide on polycrystalline gold, an additional optical absorption induced by bromine was reported ${ }^{52}$.

Image states at a silver (111) surface still exist after coverage with one monolayer of $\mathrm{Xe}^{53}$. Since electron emission from metals into aqueous electrolytes at $0 \mathrm{~V}$ NHE sets in at a photon energy of $3.23 \mathrm{eV}^{54}$, we see no reason why short-lived image states do not exist at metallic electrodes. The alternative process involving transitions between filled surface states and unfilled bulk states is, of course, not influenced by the fate of the image states.

A significant decrease in the surface susceptibility is to be expected when the electronic states at the surface become depleted by a shift above $\mathrm{E}_{\mathrm{F}}$. Exampes of this effect may be found in the following two observations:

(1) The quenching of SERS by oxygen was assigned in Ref. 55 to the "passivation of sites of increased surfaceelectron-photon" coupling by adsorption of atomic oxygen at these sites and consequently the transfer of electron density at $E_{F}$ into the $02 p$ orbitals, $2.9 \mathrm{eV}$ below $E_{F}$ as observed by photoemission ${ }^{56}$. In the sense of the model in the previous section the notion of "sites of increased surface-electron-photon coupling" corresponds to the possibility of exciting intraband transitions in surface states by the availability of elastic scattering by surface roughness. Given the density, $n$, of electrons in surface states estimated above, an $\mathrm{O}^{-\mathrm{x}}$ atom at the surface can deplete an area corresponding to $\mathrm{x} / \mathrm{n}$ surface unit cells. This is the "passivation". The only exception to this is the optical charge transfer transition of an electron from the $\mathrm{O} 2 \mathrm{p}$ orbital back towards the depleted surface state. Therefore, the only SER signal which is not quenched by atomic oxygen is the O-metal vibration (see Fig. 2). We see an analogy to measurements by Pettinger et al. ${ }^{57}$ of oxygen species at reconstructed $\mathrm{Ag}(111)$ : the Raman signal of the subsurface and bulk oxygen species, $\mathrm{O}_{\gamma}$, is quenched by the adsorbed oxygen species, $\mathrm{O}_{\beta}$.

(2) Gao and Lopez-Rios have observed the quenching of SERS of cold-deposited silver by approximately a monolayer coverage of palladium ${ }^{58}$. Palladium (111) has the 
crystal-induced surface state at about $1.3 \mathrm{eV}$ above $\mathrm{E}_{\mathrm{F}}$ (see Ref. 59). When approximately a monolayer of Ag was deposited on top of the Pd layer, the "SERS activity" was recovered.

Of-course, the results, interpretations, and conjectures of this work will allow further development of a model of the electronic contribution to surface enhanced Raman scattering (SERS) ${ }^{55}$, especially concerning the surfaceelectron-photon coupling.

\section{References}

1. Roy, D.; Furtak, T.E. Chem. Phys. Lett. 1986, 124 299.

2. Pockrand, I.; Otto, A. Solid State Commun. 1981, 38 1159.

3. Kamitakahara, W.A.; Brockhouse, B.N. Phys. Lett. 1969, 29A 639.

4. Pockrand, I.; Otto, A. Solid State Commun. 1981, 37 109.

5. Regis, A.; Dumas, P.; Corset, J. Chem. Phys. Lett. 1984, 107502.

6. Roy, D.; Furtak, T.E. Phys. Rev. B 1986, 345111.

7. Akemann, W.; Otto, A. Surf. Sci. 1992, 272211.

8. Akemann, W.; Otto, A. Phil. Mag. B 1994, 70747.

9. Macomber, S.H.; Furtak, T.E. Solid State Commun. 1983, 45267.

10. Campbell, C.T. Surf. Sci. 1985, 15743.

11. Pettenkofer, C.; Pockrand, I.; Otto, A. Surf. Science 1983, 13552.

12. Akemann, W.; Otto, A. J. Raman Spectrosc. 1991, 22 797.

13. Akemannn, W.; Otto, A.H. Schober, to be published.

14. Backeshoff, E. Diplomarbeit, Universität Düsseldorf 1986.

15. Schober, H.R.; Dederichs, P.H.; Landolt-Börnstein NS III, 13a, Springer: Berlin 1981.

16. Wetzel, H.; Gerischer, H.; Pettinger, B. Chem. Phys. Lett. 1981, 78392.

17. Otto, A. In Light Scattering in Solids, Vol. IV, ed. by M. Cardona, G. Güntherodt, Springer 1984.

18. Ertürk, Ü.; Pettenkofer, C.; Otto, A. J. Electron Spectrosc. Relat. Phenom. 1986, 38113.

19. Electronic Surface and Interface States on Metallic Systems, ed. E. Bertel, M. Donath, World Scientific 1995.

20. Bertel, E. In Ref. 18.

21. Heimann, P.; Neddermeyer, H.; Roloff. H.F. J. Phys. 1977, C10 L17.

22. Hsieh, T.C.; Miller, T.; Chiang, T.C. Phys. Rev. Lett. 1985, 552483.

23. Kevan, S.D.; Eberhardt, W. In Angle Resolved Photoemission, Studies in Surface Science and Catalysis 74 ed. by S.D. Kevan, (Elsevier 1992).

24. Avouris, Ph.; Lyo, I-W. Science 1994, 264942.
25. Ortega, J.E.; Himpsel, F.J.; Haight, R.; Peale, R.D. Phys. Rev. B 1994, 4913859.

26. Jung, T.; Mo, Y.W.; Himpsel, F.J. Phys. Rev. Lett. 1995, 741641.

27. Fischer, R.; Fauster, T.H.; Steinmann, W. Phys. Rev. B 1993, 4815496 Th. Fauster, Appl. Phys. A 1994, 59 479.

28. Giessen, K.; Hage, F.; Himpsel, F.J.; Rieß, H.J.; Steinmann, W. Phys. Rev. Lett. 1985, 55300.

29. Th. Fauster, private communication.

30. Goldmann, A. In Ref 18.

31. Giessen, K.; Hage, F.; Himpsel, F.J.; Riess, H.J.; Steinmann, W. Phys. Rev. 1986, B 335241.

32. Cardona, M. In Light Scattering in Solids II, Cardona, M.; Güntherodt, G. Eds. Springer 1982, chapter 2.

33. Drexel, W. Z. Physik 1972, 255281.

34. Gilat, G.; Raubenheimer, L.J. Phys. Rev. 1966, 144 390.

35. Paniago, R.; Matzdorf, R.; Meister, G.; Goldmann, A. Surf. Sci. 1995, 336113.

36. A filled surface state on $\mathrm{Ag}(110)$ at the $\mathrm{Y}$ point less than $0.1 \mathrm{eV}$ below the Fermi energy has been observed by Bartynski, R.A.; Gustafsson, T. Phys. Rev. B 1986, 33 6588. On $\operatorname{Ag}(100)$ a filled surface state at the $\mathrm{Ag}(100)$ surface may exist at the X-point, see Kolb, D.M.; Boeck, W.; Ho, K.-M.; Liu, S.H. Phys. Rev. Letters 1981, 471921.

37. Graß, M.; Braun, J.; Borstel, G.; Schneider, R.; Dürr, H.; Fauster, Th.; Dose, V. J. Phys.: Condens. Matter $1993,5599$.

38. Schober, H. R., unpublished results.

39. Urbach, L.E.; Percival, K.L.; Hicks, J.M.; Plummer, E.W.; Dai, H.-L. Phys. Rev. B 1992, 453769.

40. Doak, R.B.; Harten, U.; Toennies, J.P. Phys. Rev. Letters 1983, 51578.

41. Harten, U.; Toennies, J.P.; Wöll, Ch. Faraday Discuss. Chem. Soc: 1985, 80137.

42. Akemann, W.; Otto, A. Electrochimica Acta 1989, 34 35.

43. Eberhardt, W.; Plummer, E.W. Phys. Rev. $B$ 1983, 28 3605.

44. Kleinherbers, K.K.; Goldmann, A. Surf. Science 1985, 154489

45. Salaita, C.N.; Lu, F.; Laguren-Davidson, L.; Hubbard, A.T. J. Electroanal. Chem. 1987, 2291.

46. Magnussen, O.M.; Ocko, B.M.; Adzic, R.R.; Wang, J.X. Phys. Rev. 1995, 515510.

47. Valette, G.; Hamelin, A.; Parsons, R. Z. Phys. Chem. (NF) 1978, $1 / 371$.

48. Winkes, H. Dissertation Düsseldorf 1996, H. Winkes et al., to be published.

49. Newns, D.M. Phys. Rev. 1969, 1781123. 
50. Pettinger, B.; Philpott, M.R.; Gordon II, J.G. J. Phys. Chem. 1981, 852746.

51. Owen, J.F.; Chen, T.T.; Chang, R.R.; Laube, B.L. Surface Science 1983, 125679.

52. Adzic, R.; Yeager, E.; Cahan, B.D. J. Electroanal. Chem. 1977, 85267.

53. Merry, W.R.; Jordan, R.E.; Padowitz, D.F.; Harris, C.B. Surf. Science 1993, 295393.

54. Furtak, T.E.; Kliewer, K.L. Comments Solid State Phys. 1982, 10103.

55. Otto, A.; Mrozek, I.; Grabhorn, H.; Akemann, W. J. Phys. Condens. Matter 1992, 41143.
56. Eickmans, J.; Otto, A.; Goldmann, A. Surf. Science 1985, 149293.

57. Pettinger, B.; Bao, X.; Wilcock, I.C.; Muhler, M.; Ertl, G. Phys. Rev. Lett. 1994, 721561.

58. Gao, Y.; Lopez-Rios, T. Phys. Rev. Lett. 1984, 53 2583.

59. Fauster, Th.; Steinmann, W. In Electromagnetic Waves: Recent Developments in Research, Vol. 2: Photonic Probes at Surfaces, ed. by Paul Halevi, Elsevier 1995.

60. Goldmann, A.; Dose, V.; Borstel, G. Phys. Rev. B $1985,321971$.

61. Goldmann, A. In Ref 19. 